\title{
Usability Guidelines for Mobile Devices: Length of Hyperlinks
}

\author{
Eva Garcia-Lopez, Antonio Garcia-Cabot, Luis de-Marcos, and Jose-Ramon Hilera \\ Computer Science Department, University of Alcalá, \\ Ctra. Madrid-Barcelona Km 33.6, 28871, Alcalá de Henares, Madrid, Spain \\ \{eva.garcial, a.garciac, luis.demarcos, jose.hilera\}@uah.es
}

\begin{abstract}
The use of mobile devices is increasing in recent years, and their characteristics are different from those of computers (keyboard and screen size, interaction method, etc.), so it is important to specifically study the usability of these devices. This paper presents an experiment carried out with users to find the correct length (from the point of view of usability) of hyperlinks in mobile devices. A real mobile device has been used for the experiment, as well as more than 20 users. The experiment compared three different lengths of hyperlinks and it concluded that users prefer hyperlinks as short as possible and they are identified better when the text is not justified.
\end{abstract}

Keywords: Hyperlinks, usability, guidelines, mobile devices.

\section{Introduction}

The rise of mobile devices has led to a transformation in the way of creating digital content, not just web pages but any content that these devices can play [1]. Some characteristics of these devices, such as the small screen size or the way of interaction, pose a real challenge when generating this content for mobile devices [2].

Focusing on web content development from the point of view of usability, there are several efforts to establish guidelines and/or recommendations to generate this content in a usable way [3].

Some important aspects of the web, by its nature, are hyperlinks that allow linking some content with another one. From the point of view of traditional computers, there have been many efforts to establish guidelines and recommendations to create these hyperlinks (their size, significance, etc.); but from the point of view of web content for mobile devices, perhaps these guidelines cannot be used or should be adapted by taking into account the limitations of these devices.

Therefore, this paper presents a solution to the problem of which length should hyperlinks have when they are shown in mobile devices, always from a usability perspective.

To solve the problem, this paper presents a set of experiments carried out with users and with different kinds of hyperlinks that allow figuring out the right size for them. 
Section 2 of this paper shows the background and the related work to this research, Section 3 explains the details of the experiments carried out, Section 4 presents the results and finally Section 5 shows the conclusions and future work.

\section{Background and Related Work}

Some guidelines and standards establish a concrete length for hyperlinks. In some cases the link length is not explicitly mentioned, but a link should be understandable enough, regardless of its length. Furthermore, some of these guidelines were established for web pages in general, such as [4], [5]. However, other guidelines have already been defined specifically for mobile devices [6], [7].

The standard ISO 9241-151 [4], designed specifically for general purpose web pages, states in guideline 9.4.13 "Link length" that "Textual link names should be long enough to be understood but short enough to avoid wrapping".

The initiative Usability.gov [5] states in guideline 10:11 "Use Appropriate Text Link Lengths" the following: "Make text links long enough to be understood, but short enough to minimize wrapping", which somewhat matches the ISO guideline about creating links long enough to be understood but preventing wrapping.

Furthermore, Budiu, R. and Nielsen, J. [6] propose a set of guidelines focused on web and applications design for mobile devices, and in guideline 103 they state "Use links with good information scent (that is, links which clearly indicate where they take the users) on your mobile pages". The length that links should have is not explicitly shown, but it indicates they must be clear and descriptive.

Finally, W3C [7] has best practices for creating web pages, and guideline 5.2.6 "Link Target Identification" states that links should clearly indicate the target of the link, but does not mention any length that links should have.

Due to the above, this paper presents a set of experiments with real users to help making a recommendation about the importance of the size of hyperlinks in mobile devices. The hypothesis will be taken from the aforementioned guidelines of ISO 9241-151 and Usability.gov, i.e., "Texts of the links should be long enough to be understood and short enough to avoid wrapping" because, although they are not guidelines applied directly to mobile devices, they are concrete and specific.

\section{Planning the Experiment}

An experiment was designed to test the usability of different link lengths using metrics from the "usability" concept according to ISO 9241-11 [8] (effectiveness, efficiency and satisfaction). For this, three types of web pages (Fig. 1.) were designed, each of which contained a text with some words or sentences marked as links. These links were colored and underlined, as suggested by [9], to maximize the perceived affordance of clickability. The text used was extracted from a chapter of The Theory of Evolution by Charles Darwin.

Links in the first web page were short, i.e., each one of them consisted of a single word. In the second web page links were medium, i.e., they had at least two words but without taking up more than two lines. Finally, links in the third web page were long, occupying several lines. 

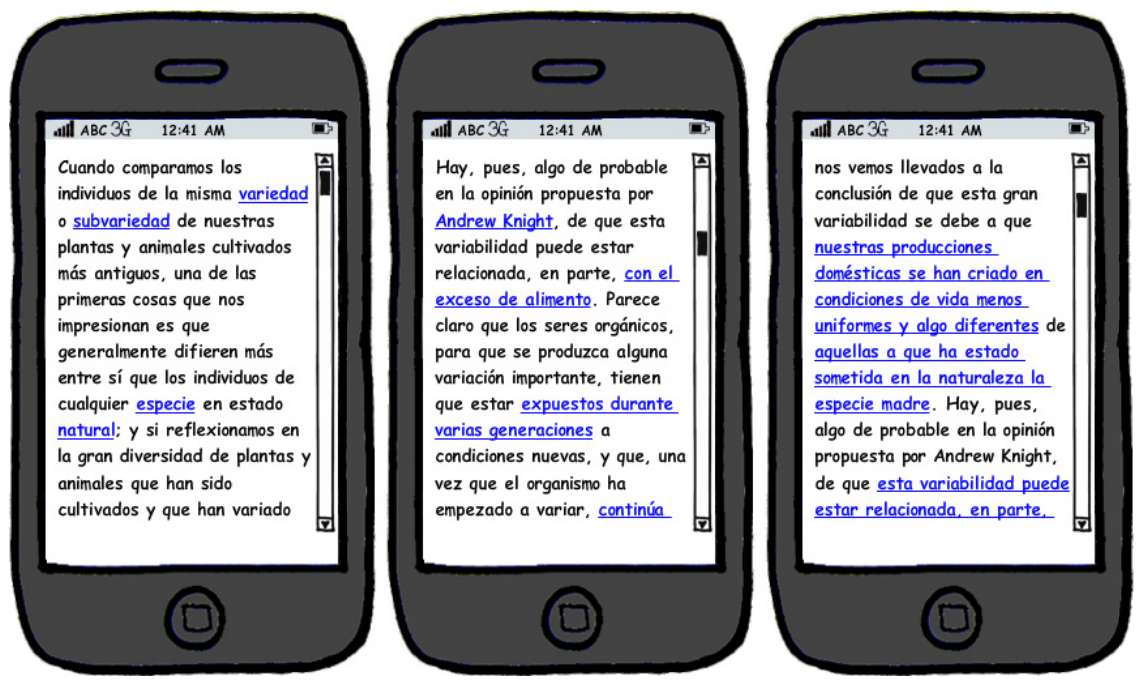

Fig. 1. Prototype of web pages designed for the experiment: short, medium and long size links, respectively (in Spanish)

Users were asked to count the number of links in each of the three web pages, so that the effectiveness could be measured objectively, using the following formula:

$$
\text { effectiveness }=1-\frac{|n-i|}{n}
$$

where $n$ is the number of real links and $i$ is the number of links the user counted.

It is noteworthy that the links were disabled, that is, if the user clicked on it, no action would be triggered. This is to prevent the user found out if, for example, a long link was composed of a single link or more than one.

Another parameter to measure was the time that users took to count the number of links. In this way, efficiency could be measured, taking into account the average time taken in counting the number of links in each of the cases.

Finally, after the experiment, users completed a satisfaction survey in which they were asked about how comfortable was for them the act of reading the text with each type of links, and if the target of the links was easily understood in each web page of the experiment. The survey had also some "free response" questions about the opinion of each web page, so we could infer what users think, in general, about each of the types of links (short, medium and long size).

In order that the text of the web page was not the same in all treatments, three different versions of web pages were designed with the three types of links in each, so that there were nine different web pages for distribution to users. The number of links for each web page was different. Treatments were randomly distributed among users, so that each user performed the experiment with a web page with short links, another one with medium size links and finally one with long size links, but never with the same text. 
Additionally, other three web pages were created with the same text and with different links (long size), but with justified text instead of left-aligned. This is because when the experiment was performed with "subject 0" (the first user that participated in the experiment), we realized that the justification (or not) of text could confuse the user when counting the links, especially when they take up more than one line, as it is the case of long size links.

\section{$4 \quad$ Results and Discussion}

The experiment was performed with 22 subjects, of which $77.27 \%$ were male and $22.73 \%$ female. $63.64 \%$ of the subjects were between 18 and 24 years; $22.73 \%$ were between 25 and 34 years; and $13.64 \%$ were older than 35 years. Of these, $45.45 \%$ was considered expert in the use of mobile devices, $45.45 \%$ was considered in an intermediate level, and $9.09 \%$ was considered novice. Regarding the highest level of education completed, $13.64 \%$ of the subjects had High School completed and were studying a Degree; $31.82 \%$ had Associated Degree; $18.18 \%$ had a Degree; $31.82 \%$ had a Master Degree and $4.55 \%$ had a $\mathrm{PhD}$.

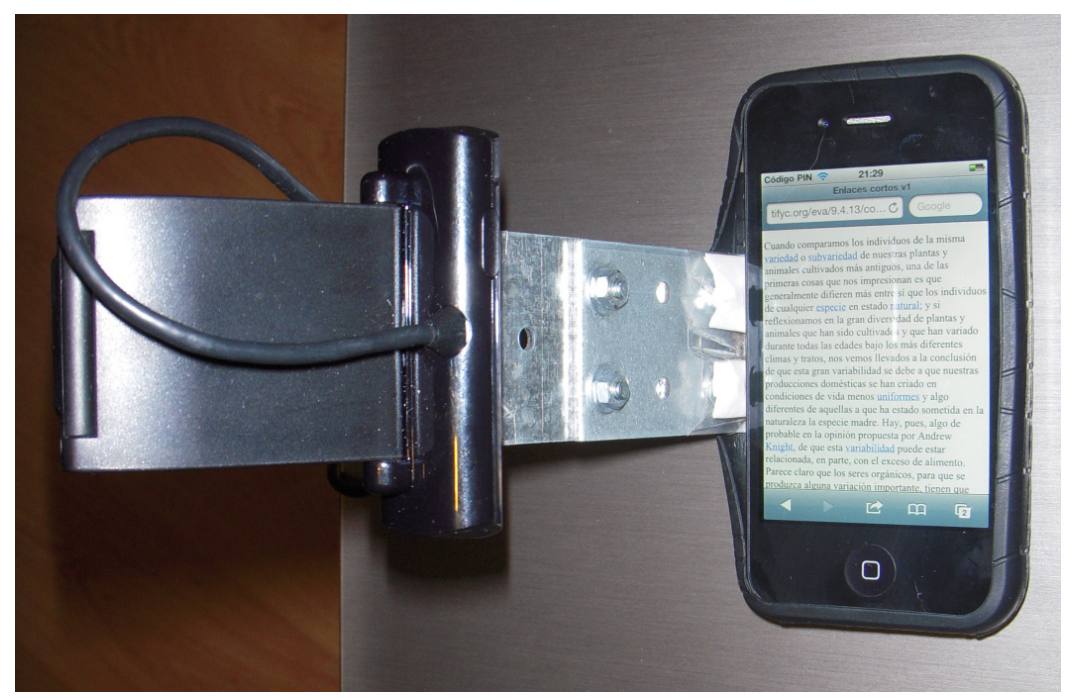

Fig. 2. Mechanism used to record user interactions

To carry out the experiment, a mobile device of make Apple was used (specifically model iPhone 4) and also a webcam that recorded continuously the screen that the user was seeing. The webcam was joined to a metal structure, and the structure was joined to a soft housing specific for this kind of device. The webcam was connected to a laptop via the USB cable, so the videos were recorded directly into the laptop. 
Four videos were recorded for each user, one for each factor:

- One with unjustified text and short size links.

- Another with unjustified text and medium size links.

- Another one with unjustified text and long size links.

- Another one with justified text and long size links.

To measure effectiveness, as mentioned above, it was measured by the percentage of correct answers of the subjects to count the number of links in each configuration, that is, with short size links, with medium size links, with long size links and unjustified text, and with long size links and justified text. Then the average of successes of all subjects for each configuration and the standard deviation were obtained (Table 1).

Table 1. Results obtained for effectiveness

\begin{tabular}{lccc}
\hline Type of link & Average $(\%$ Success) & Standard Deviation & Median \\
\hline Short size & 99.68 & 0.0152 & 1 \\
Medium size & 100 & 0.0000 & 1 \\
Long size and unjustified text & 98.82 & 0.0312 & 1 \\
Long size and justified text & 97.30 & 0.0465 & 1 \\
\hline
\end{tabular}

Later a normality test for data was executed, in order to determine the most appropriate method of analysis (Table 2).

Table 2. Normality test for effectiveness

\begin{tabular}{lc}
\hline Type of link & $p$ \\
\hline Short size & $<0.005$ \\
Medium size & NA* \\
Long size and unjustified text & $<0.005$ \\
Long size and justified text & $<0.005$ \\
\hline
\end{tabular}

*All values are 1 (100\% success) for medium-sized links so $p$ cannot be calculated.

Data did not pass the normality test $(p<0.05)$ so nonparametric methods should be used. Kruskal-Wallis test returns $p=0.009(<0.05 ; \mathrm{H}(3)=11.46)$, so the results are statistically significant. That is, the size of the links affects the effectiveness. To distinguish those groups that are statistically better and worse than others, another KruskalWallis test was applied, but this time by pairs of groups (Table 3 ).

Table 3. Results of Kruskal-Wallis test

\begin{tabular}{lcc}
\hline Type of link & $p$ & $\mathrm{H}$ \\
\hline Short and medium sized links & 0.317 & 1 \\
Short links and long links with unjustified text & 0.290 & 1.12 \\
Short links and long links with justified text & 0.022 & 5.23 \\
Medium links and long links with unjustified text & 0.076 & 3.14 \\
Medium links and long links with justified text & 0.004 & 8.07 \\
Long links with unjustified text and long links with justified text & 0.195 & 1.68 \\
\hline
\end{tabular}


In view of the results, short and medium sized links are statistically more effective (i.e., users make fewer mistakes) than long links with justified text. Moreover, one can say, with $90 \%$ confidence, that medium sized links are statistically more effective than long links with unjustified text.

Furthermore, for efficiency, we analyzed the time each subject took to count the number of links with each configuration, and the average, standard deviation and median were obtained for each (Table 4).

Table 4. Results obtained for efficiency

\begin{tabular}{lccc}
\hline Type of link & Average (seconds) & Standard Deviation & Median \\
\hline Short links & 14.091 & 6.286 & 12 \\
Medium links & 15.773 & 6.164 & 13 \\
Long links and unjustified text & 26.455 & 12.054 & 21 \\
Long links and justified text & 23.273 & 7.857 & 22 \\
\hline
\end{tabular}

First of all, a normality test was applied to data, in order to determine the most adequate analysis method (Table 5).

Table 5. Normality test for efficiency

\begin{tabular}{lc}
\hline Type of link & $p$ \\
\hline Short links & $<0.005$ \\
Medium links & 0.009 \\
Long links and unjustified text & $<0.005$ \\
Long links and justified text & 0.222 \\
\hline
\end{tabular}

Data did not pass the normality test $(p<0.05)$, so nonparametric methods should be used. Kruskal-Wallis test returns $p=0.000(<0.05, \mathrm{H}(3)=29.53)$, from which it follows that the results are statistically significant, i.e., short and medium sized links are statistically more efficient (faster to identify) than long size links. It is not possible to say that there is statistical significance between short and medium sized links; nor between long links with unjustified text or with justified text, so it cannot be said that some are more efficient than others.

Finally, the surveys that users answered after the experiment were analyzed to know the satisfaction of the users. Answers to three questions were assessed, which were the following for each configuration:

- Q1: Is it comfortable to read the text?

- Q2: Is the target of the link easy to understand?

- Q3: What do you think, in general, you will get if you click on the links?

The answers to the first two questions were measured quantitatively on a Likert scale from 0 to 5 . The results for each configuration (average of answers of all users and standard deviation) are shown in Table 6. 
Table 6. Survey results

\begin{tabular}{lcccc}
\hline Type of link & $\begin{array}{c}\text { Q1 } \\
\text { (Average, } \\
\text { from 0 to 5) }\end{array}$ & $\begin{array}{c}\text { Q1 } \\
\text { (Standard } \\
\text { deviation) }\end{array}$ & $\begin{array}{c}\text { Q2 } \\
\text { (Average, } \\
\text { from 0 to 5) }\end{array}$ & $\begin{array}{c}\text { Q2 } \\
\text { (Standard } \\
\text { deviation) }\end{array}$ \\
\hline Short links & 4.36 & 0.726 & 3.27 & 1.579 \\
Medium links & 3.27 & 1.077 & 3.05 & 1.090 \\
Long links and unjustified text & 1.41 & 1.007 & 2.36 & 1.733 \\
Long links and justified text & 1.55 & 1.299 & 2.59 & 1.436 \\
\hline
\end{tabular}

As for the third question, answers of the subjects were "free", so it is difficult to quantify. However, much of the users $(90.91 \%)$ think that short links will lead to a web page where the definition of the word is explained, while long and medium sized links usually make to think (by $77.27 \%$ of cases) that will lead to a web page that explains in more detail the link sentence.

\section{$5 \quad$ Conclusions and Future Work}

In view of the results above, the following conclusions are obtained:

- Looking at the comfort for reading a text of a web page from a mobile device (Q1), there is no significant different between justifying or do not the text, that is, some users think that reading the text is more comfortable when it is justified but some others think it is easier when the text is not justified, since there is no a big difference between their averages. However, efficiency is influenced negatively by the text justification, i.e., when the text is justified, users seem unable to differentiate where a link starts and where it ends as well as when the text is not justified. Therefore, one could argue that it is preferable do not justify the text so that the user can clearly distinguish where the links start and end, although this affirmation is based on the average of data and it must be taken into account that it is not statistically significant.

- Users prefer clearly short links (according to Q1 average), because they think that is the most comfortable way to read a text containing links. Medium links are not as uncomfortable as long links, and observing an increasing score in reading comfort from long links to short links, it follows that users seem to prefer links as short as possible. This conclusion is further supported because data suggest that users often understand better the target of a link (according to Q2 average) the shorter (there is an increasing score about understanding the target of the link, from the longest to the shortest), and also because effectiveness is higher the shorter the link (users seem to spend less time when identifying the links). As a remark, note that the effectiveness is slightly better with medium links than with short links, but this does not really matter, since only one user was confused when he counted short links. Therefore, the conclusion "the shorter the links the better" is still valid despite this fact. 
- Comparing the above conclusions with the initial hypothesis ("Texts of the links should be long enough to be understood and short enough to avoid wrapping"), one could say that in the case of mobile devices this is not true because users seem to prefer links as short as possible, and also the data suggest that they are better and faster identified, both where they start and end, and their target. Therefore, a possible recommendation about the link length in web pages for mobile devices would be: "Texts of the links should be as short as possible and, whenever possible, the text in which they are contained should not be justified".

For future work, the experiment could be repeated with different mobile devices with diverse characteristics (techniques of interaction, screen size, screen orientation), in order to check if conclusions can be extrapolated to other devices aside from smartphones. Also, in addition to the length, the semantics of links should be considered to evaluate the understanding of the links by users.

Acknowledgement. This research has been co-funded by the Master in Software Engineering for the Web of the University of Alcalá, the projects IPT-2011-0795430000 and UAH2011/6XP-0043090XT099, and the TIFyC research group.

\section{References}

1. Gómez, S., Fabregat, R.: Context-Aware Content Adaptation in mLearning. In: Montebello, M., Camilleri, V., Dingli, A. (eds.) 9th World Conference on Mobile and Contextual Learning, MLEARN 2010, pp. 76-83 (2010)

2. Zhang, D., Adipat, B.: Challenges, Methodologies, and Issues in the Usability Testing of Mobile Applications. International Journal of Human-Computer Interaction 18(3), 293-308 (2005)

3. Nielsen, J., Budiu, R.: Mobile usability. New Riders, Berkeley (2012)

4. ISO. ISO 9241-151:2008. Ergonomics of human-system interaction - Part 151: Guidance on World Wide Web user interfaces (2008)

5. U.S. Department of Health and Human Services'. Research-Based Web Design and Usability Guidelines (2002), http://usability.gov/guidelines (last accessed March 22, 2013)

6. Budiu, R., Nielsen, J.: Usability of Mobile Websites and Applications, Design Guidelines for Improving the User Experience of Mobile Sites and Apps. Report, Nielsen Norman Group (2011)

7. W3C. W3C Mobile Web Best Practices 1.0 (2008), http: / /www.w3 . org/TR/mobile-bp/ (last accessed March 22, 2013)

8. ISO. ISO 9241-11:1998. Ergonomic requirements for office work with visual dis-play terminals (VDTs) - Part 11: Guidance on Usability (1998)

9. Nielsen, J.: Jakob's Nielsen Alertbox: Guidelines for Visualizing Links (2004), http://www.nngroup.com/articles/guidelines-forvisualizing-links / (last accessed March 24, 2013) 